\title{
Fusarium Head Blight Modifies Fungal Endophytic Communities During Infection of Wheat Spikes
}

\author{
Edward C. Rojas $^{1}$ (I) $\cdot$ Rumakanta Sapkota ${ }^{2}$ (I) $\cdot$ Birgit Jensen $^{1}$ (D) - Hans J. L. Jørgensen ${ }^{3}$ (D) Tina Henriksson $^{4}$. \\ Lise Nistrup Jørgensen ${ }^{2} \cdot$ Mogens Nicolaisen $^{2}$ (D) - David B. Collinge ${ }^{1}$ (C)
}

Received: 23 November 2018 / Accepted: 13 August 2019 / Published online: 26 August 2019

(C) The Author(s) 2019

\begin{abstract}
Fusarium head blight (FHB) is a devastating disease of wheat heads. It is caused by several species from the genus Fusarium. Several endophytic fungi also colonize wheat spikes asymptomatically. Pathogenic and commensal fungi share and compete for the same niche and thereby influence plant performance. Understanding the natural dynamics of the fungal community and how the pre-established species react to pathogen attack can provide useful information on the disease biology and the potential use of some of these endophytic organisms in disease control strategies. Fungal community composition was assessed during anthesis as well as during FHB attack in wheat spikes during 2016 and 2017 in two locations. Community metabarcoding revealed that endophyte communities are dominated by basidiomycete yeasts before anthesis and shift towards a more opportunistic ascomycete-rich community during kernel development. These dynamics are interrupted when Fusarium spp. colonize wheat spikes. The Fusarium pathogens appear to exclude other fungi from floral tissues as they are associated with a reduction in community diversity, especially in the kernel which they colonize rapidly. Similarly, the presence of several endophytes was negatively correlated with Fusarium spp. and linked with spikes that stayed healthy despite exposure to the pathogen. These endophytes belonged to the genera Cladosporium, Itersonillia and Holtermanniella. These findings support the hypothesis that some naturally occurring endophytes could outcompete or prevent FHB and represent a source of potential biological control agents in wheat.
\end{abstract}

Keywords Fusarium graminearum $\cdot$ FHB $\cdot$ Fungal communities $\cdot$ Endophytes $\cdot$ Metabarcoding

Electronic supplementary material The online version of this article (https://doi.org/10.1007/s00248-019-01426-3) contains supplementary material, which is available to authorized users.

Edward C. Rojas

e.tayo@plen.ku.dk

1 Section for Microbial Ecology and Biotechnology, Department of Plant and Environmental Sciences \& Copenhagen Plant Science Centre, University of Copenhagen, Thorvaldsensvej 40, DK-1871 Frederiksberg C, Denmark

2 Department of Agroecology, Faculty of Science and Technology, Aarhus University, Forsøgsvej 1, DK-4200 Slagelse, Denmark

3 Section for Plant and Soil Science, Department of Plant and Environmental Sciences \& Copenhagen Plant Science Centre, University of Copenhagen, Thorvaldsensvej 40, DK-1871 Frederiksberg C, Denmark

4 Lantmännen Lantbruk, Onsjövägen 13, 26831 Svalöv, Sweden

\section{Introduction}

Wheat (Triticum aestivum) is one of the most important crops in the world. Global production reaches around 750 million metric tons annually and it is a key cereal crop for human food supply [1]. Wheat productivity is vulnerable to diseases caused by fungal pathogens [2]. Fusarium head blight (FHB) is an economically important disease in wheat and other cereal crops [3]. It is caused by several species in the genus Fusarium, among which $F$. graminearum, F. culmorum, F. poae, $F$. avenaceum, $F$. langsethiae and $F$. sporotrichioides are generally considered the most important in Europe [4, 5]. FHB causes substantial yield losses by infecting and colonizing the spikes and the kernel during anthesis. The pathogens cause bleached lesions on the glumes and rachis and damage the grain [6]. More importantly, they produce specialized metabolites including trichothecenes and zearalenone (mycotoxins) [7]. These molecules facilitate infection and have been shown to play a role in competition with other microorganisms [8], but 
also reduce grain quality for consumption as they can cause health problems to humans and animals [9].

However, pathogens are not the only microorganisms colonizing plant tissues. Wheat is colonized by a highly diverse array of microorganisms including other fungal species, some of which live asymptomatically during their life within the plant [10]. These communities of endophytic organisms (endophytes) appear to be ubiquitous among species in the kingdom Plantae and are thought to interact closely with the plant as commensals or symbionts [11]. The importance of these communities in plant fitness has emerged in recent years [12] and certain of these fungi or whole communities in concert are considered to be involved in increased tolerance to stress $[13,14]$ and competition with pathogens [15].

Since these microorganisms possess similar characteristics and share essentially the same biological niche as pathogens, it is relevant to understand their relationships and behaviour during disease outbreaks [16]. Moreover, some of these microorganisms may have the potential to be exploited in disease control as biological alternatives or to complement current control options [17]. If fungal endophytes were to be exploited as a new source of biocontrol agents, it would necessitate a thorough understanding of their behaviour when the pathogen arrives and the disease occurs [18].

The main objective of this study was to assess the overall composition of the fungal endophytic community inside wheat spikes at the flowering stage and during FHB attack. Additionally, we aimed to detect endophytic species that could be associated to naturally occurring biological control. To our knowledge, this is the first report determining the endophytic fungal microbiome of different tissues during wheat spike development as well as the first analysis of the composition of these communities under FHB attack. These results reveal the magnitude of the pathogen-associated changes on the plant microbiome and support the concept that endophytic fungi may represent a new source of biocontrol agents against plant diseases.

\section{Materials and Methods}

\section{Field Conditions}

Trials were located at Flakkebjerg, Denmark (Aarhus University field station), $55^{\circ} 19^{\prime} 31.6^{\prime} \mathrm{N} 11^{\circ} 23^{\prime} 01.7^{\prime} \mathrm{E}$ and at

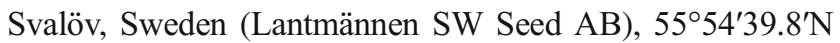
$13^{\circ} 07^{\prime} 09.3^{\prime} \mathrm{E}$. In both locations, multiple winter wheat cultivars were tested for their response to FHB, under optimal infection conditions, for two consecutive years (2016 and 2017). Cultivars were grown in randomized $1 \mathrm{~m}^{2}$ plots and each cultivar had four replications. A diagram of the trial layout is shown in Supplementary Fig. 1.

\section{Fusarium spp. Inoculation}

Trials were artificially infected with a mixture of Fusarium spp. (mainly $F$. graminearum and F. culmorum isolates from the area). A list of the species and isolates used at each location can be seen in Supplementary Table 1. In Denmark, spikes were sprayed directly with a Fusarium spp. macroconidial suspension $\left(2 \times 10^{6}\right.$ spores $\left./ \mathrm{ml}, 5 \mathrm{ml} / \mathrm{m}^{2}\right)$ three times during anthesis (BBCH 60, 65 and 69, [19]) and irrigation was provided before and after inoculation. In Sweden, Fusarium spp. isolates were cultured on sterilized wheat and oat grains (imbibed for $16 \mathrm{~h}$ before inoculation) for 36 days. Kernels were then dried on paper towels for 4 days in a greenhouse. Once dried, the kernels were spread between plants before the flowering stage (BBCH 60) at a dose of $30 \mathrm{~g} / \mathrm{m}^{2}$ and irrigation was not provided.

\section{Cultivar Selection and Sampling}

FHB severity was assessed at 14 days after inoculation (BBCH 85). A visual linear scale from 0 to 10 was used to assess the percentage of infected plants within each plot (each level representing $10 \%$ increase in disease severity). A value was given to each plot and the mean of the four replications was calculated to determine infection severity for each cultivar. One cultivar was selected from each location during the first year, and the same cultivar was used during the second year. Cultivars KW-Nils and SW 14308 were selected for sampling in Denmark and Sweden, respectively, due to their low infection levels based on the visual assessment during the first year (5 and $13 \%$, respectively). Severity for these two cultivars during the second year was $42 \%$ for KWS-Nils (Denmark) and 38\% for SW14308 (Sweden). Spike sampling was performed at BBCH 90. In each of the four plots, four spikes with FHB symptoms ('FHB-symptoms') and four spikes with no symptoms ('Symptomless') were collected in order to obtain 16 spikes in for each Fusarium-status (FHBsymptoms or Symptomless). Spikes were selected throughout the plots avoiding the margins.

Additionally, 16 healthy spikes from the same cultivars growing in a non-inoculated adjacent plot were collected as a non-inoculated control (Fusarium-status: 'Control'), see Supplementary Fig. 1. These plants were sown on the same date and were subjected to the same agricultural practices as the inoculated plots. Sampling in these plots was performed in a randomized way avoiding the margins.

During the second year, these adjacent, non-inoculated plots were also sampled at heading stage (BBCH 59) in order to assess the changes before and after anthesis in both studied location-cultivars combinations (Denmark-KWS-Nils and Sweden-SW-14308). Sixteen spikes were collected using the same sampling methodology. These spikes were designed as 
'Pre-anthesis' samples. No Fusarium infection was observed in the adjacent plots during sampling.

\section{Sample Preparation}

From each spike, five spikelets were removed from the middle section of the rachis and dissected. Glumes, lemmas and paleas were separated. Subsequently, these external bract tissues were pooled and designated as 'Bracts' independently from the kernels, designated as 'Kernel' in the analysis. Both types of tissues were surfaced sterilized using $96 \%$ ethanol for $1 \mathrm{~min}, 2 \%$ sodium hypochlorite for $3 \mathrm{~min}$ and $96 \%$ ethanol for $30 \mathrm{~s}$ followed by rinsing twice with sterile Milli-Q water. A volume of $100 \mu \mathrm{l}$ from the last rinsing water was plated on potato dextrose agar plates (Difco ${ }^{\mathrm{TM}}$ ) to assess the efficiency of the surface sterilization. Tissue types ('Bracts', 'Kernels') from three spikes were pooled as one replication. Four replications for each Fusarium-status (FHB-symptoms, Symptomless, Control), tissue type (Bracts, Kernels) and location-cultivar (Sweden: cv. KWS-Nils, Denmark: cv. SW14308) were prepared for the first year (samples first year 48). During the second year, five replications were prepared for each group plus an additional condition: 'Pre-anthesis' samples (samples second year 80). Increment in the number of replications from year 1 to year 2 was due to availability of a fifth plot for the cultivars of interest during the second year (extra plot was sown for the cultivars selected during the first year). In total, 128 samples were prepared for downstream analysis.

\section{DNA Extraction and Library Preparation}

Samples were freeze dried for $48 \mathrm{~h}$ and homogenized using ceramic beads $(10 \mathrm{~mm})$ in a mechanical shaker (SO-40a, Fluid Management Europe B.V.) using a 30 -s programme three times. Approximately $100 \mathrm{mg}$ of homogenized tissue per sample were used for DNA extraction using the E.Z.N.A. ${ }^{\circledR}$ Plant DNA Kit Plant (Omega Bio-tek, Norcross, GA, USA), following the manufacturer's instructions. All the DNA samples were used for fungal library preparation. The fungal ITS2 region of the ribosomal RNA gene was amplified using the primers fITS7 and ITS4 [20]. Dual indexing was carried out to allow pooling of 96 samples. In addition, internal barcodes, consisting of varying numbers of nucleotides, were added to the forward primer for combining samples within each index combination $[21,22]$. Primer sequences with internal barcodes and the index combinations are shown in Supplementary Table 2.

Each sample was amplified three times using different internal barcodes in order to ensure higher numbers of reads per sample. PCR reactions and pooling was carried as described previously [23]. In brief, PCR was performed in a reaction mixture of $25 \mu \mathrm{l}$ consisting of the following: $5 \mu \mathrm{l}$ PCR buffer
Promega 5X (Promega Corporation, Madison, USA), $1.5 \mu \mathrm{l}$ $\mathrm{MgCl}_{2}(25 \mathrm{mM}), 2 \mu \mathrm{l}$ dNTPs ( $2.5 \mathrm{mM}$ each), $2 \mu \mathrm{l}$ of each primer $(10 \mu \mathrm{M}), 0.125 \mu \mathrm{l}(5 \mathrm{U} / \mu \mathrm{l}) \mathrm{GoTaq}{ }^{\circledR}$ Flexi polymerase (Promega Corporation, Madison, USA), $1 \mu$ DNA template

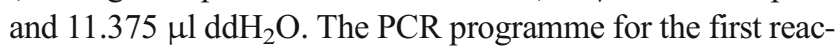
tion was $94{ }^{\circ} \mathrm{C}$ for $5 \mathrm{~min}$ followed by 30 cycles $\left(94{ }^{\circ} \mathrm{C}\right.$ for $30 \mathrm{~s}, 57^{\circ} \mathrm{C}$ for $30 \mathrm{~s}, 72^{\circ} \mathrm{C}$ for $30 \mathrm{~s}$ ) and a final elongation step at $72{ }^{\circ} \mathrm{C}$ for $10 \mathrm{~min}$. The PCR programme for the second reaction was $94{ }^{\circ} \mathrm{C}$ for $5 \mathrm{~min}$ followed by 10 cycles $\left(94{ }^{\circ} \mathrm{C}\right.$ for $30 \mathrm{~s}, 55^{\circ} \mathrm{C}$ for $30 \mathrm{~s}, 72{ }^{\circ} \mathrm{C}$ for $30 \mathrm{~s}$ ) and a final elongation step at $72{ }^{\circ} \mathrm{C}$ for $10 \mathrm{~min}$. PCR products were pooled, ethanol precipitated and re-eluted in $50 \mu \mathrm{l}$ Tris-EDTA buffer $(10 \mathrm{mM}$, $\mathrm{pH}$ 8). The pooled DNA was stained with SYBR ${ }^{\circledR}$ Green I (Sigma-Aldrich, St. Louis, USA), separated on a $1.5 \%$ agarose gel and a band of the expected size (300-450 bp) was extracted using the QIAquick® Gel Extraction Kit (Qiagen, Hilden, Germany). The concentration was measured using a NanoDrop 2000 (Thermo Scientific ${ }^{\mathrm{TM}}$ ) and determined to be higher than $100 \mathrm{ng} / \mu \mathrm{l}$. Sequencing was carried out at Eurofins MWG (Ebersberg, Germany) using the dual indexing Illumina Miseq platform. Raw read files from this study were deposited in the NCBI sequence read archive under the SRA accession: SRP184488.

\section{Bioinformatics and Statistical Analysis}

Analysis of sequence reads was performed using QIIME version 1.9 [24]. The paired end reads were joined using SeqPrep, a fasq-join tool [25] with an overlapping minimum read length of 30 base pairs. The samples were split based on indexes and pooled to a single FASTA file, while removing reads with quality Phred scores less than 20 and other default parameters using the split_libraries_fastq.py command. Chimera detection, de-replication and clustering were done using 'vsearch' version 2.6 [26]. After excluding singletons, sequences were clustered to OTUs using a $97 \%$ similarity threshold $(\geq 97 \%)$. Vsearch default options were used. Vsearch uses a distancebased greedy clustering method for clustering by default. Forward and reverse primers, internal barcodes and reads with less than $200 \mathrm{bp}$ were removed. Supplementary Table 3 includes a detailed description of the number of reads in each step of the bioinformatics process. Taxonomy assignment for the clustered operational taxonomic units (OTUs) was done using the UNITE database version 7.2 [27]. In addition, the most abundant OTUs with at least 1000 reads were also blasted against the NCBI database and taxa assigned based on top 10 hits.

All statistical analyses were carried out in $\mathrm{R}$ version 3.5.2 [28]. Diversity-based analysis was carried out using the 'vegan' [29] and 'phyloseq' [30] packages available in R. The OTU table was either transformed to relative abundance or rarefied before performing alpha and beta diversity based calculations. For beta diversity and partitioning of variance, 
Bray-Curtis dissimilarity for fungal communities was subjected to a permutational multivariate analysis of variance (PERMANOVA) using the 'adonis' test from the 'vegan' package. Alpha diversity comparisons were made using a Kruskal-Wallis one-way analysis of variance and pairwise comparisons were made using a Wilcoxon signed-rank test with false discovery rate as a $P$ value adjustment method.

Indicator species test was carried out using the DufreneLegendre Indicator Species Analysis in the 'labdsv' package [31]. Top 3 highest indicator value species under each condition were selected for Table 3. For co-occurrence networks, Spearman rank correlations among each OTU were carried out. Correlations with Spearman's rho $>0.5$ and $<-0.5$ with $P<0.05$ were considered as significantly correlated. All correlations were visualized using networks and network properties computed using the 'igraph' package [32]. All significantly correlated OTUs were used as nodes and the correlations values were used as edges.

\section{Results}

Metabarcoding of the ITS2 region using DNA from wheat spike samples from both locations for both years resulted in a total of $8,216,141$ reads. These reads clustered into 416 OTUs with $97 \%$ similarity after exclusion of non-fungal reads, using the UNITE database. A rarefaction curve indicated that sequencing coverage was successful in estimating most of the diversity in the communities (Supplementary Fig. 2). Across all samples, species from phylum Ascomycota represented $76.8 \%$ of the totally detected sequences and species from Basidiomycota $23.1 \%$. The first 69 OTUs, representing $99.5 \%$ of the total diversity, were, where possible, identified to species level using the closest BLAST hit from NCBI database. Identities, GenBank accessions, coverage and percent of identity are shown in Supplementary Table 4 . The most abundant species were Fusarium graminearum (OTU_1) and Cladosporium herbarum (OTU_2), which together represented approximately $60 \%$ of all the reads. Other abundant species were Sporobolomyces roseus (7.2\%), Vishniacozyma victoriae (6.9\%), Fusarium culmorum (4.9\%), Alternaria infectoria (3.9\%), Cryptococcus tephrensis (3\%), Aureobasidium pullulans (1.4\%) and Parastagonospora nodorum (1.4\%).

\section{Natural Community Changes During Anthesis}

Fungal communities before and after anthesis comprised mainly genera such as Cladosporium, Alternaria, Vishniacozyma and Sporobolomyces. Community changes in relative abundance between flowering time and tissue type were observed at class level. The relative abundance of basidiomycete endophytic yeasts belonging to the class
Microbotryomycetes decreased after anthesis in both 'Bracts' and 'Kernels' from nearly $40 \%$ to less than $20 \%$. Inversely, Tremellomycetes yeast increased in relative abundance in the 'Ripening' kernels from $30 \%$ to almost $50 \%$. Interestingly, 'Ripening' bracts showed a big increase in abundance of ascomycete Dothideomycetes, from nearly 25 to $50 \%$ (Fig. 1a). Community changes during flowering appeared to be associated with changes in abundance of preestablished fungal classes rather than changes in species identities.

Using three alpha diversity indices, we observed a slight increase in diversity from 'Pre-anthesis' spikes to 'Ripening' spikes. Indeed, 'Bracts' at 'Ripening' showed significantly higher Observed alpha diversity than 'Bracts' at 'Pre-anthesis' $(P<0.05)$ (Fig. 1b). Likewise, we observed that 'Kernels' harbour slightly less diversity than the 'Bracts', although no significant differences were detected. Interestingly, it was observed that spikes collected in Sweden (cv. SW 14308) showed higher diversity than those from Denmark (cv. KWNils) $(P<0.05)$ (Supplementary Fig. 3).

The fungal community structure from spikes before and after anthesis was compared using beta diversity values. PERMANOVA test showed that 'Flowering stage' (Preanthesis, Ripening) had a minor, yet significant effect on the community $\left(R^{2}=0.04, P<0.01\right)$. Additionally, 'Tissue type' (Bracts, Kernel) was highly significant $\left(R^{2}=0.07, P<0.01\right)$ just like 'Location-Cultivar' (Sweden: SW14308, Denmark: KWS-Nils) $\left(R^{2}=0.08, P<0.01\right)$. A significant interaction between 'Flowering stage' and 'Location-cultivar' was also detected $\left(R^{2}=0.08, P<0.01\right)$ (Table 1$)$. Overall, the effect of 'Flowering stage' was small and exhibited a higher diversity in the 'Bracts' at the 'Ripening' stage.

\section{FHB-Induced Community Changes}

Fungal communities in 'Control' and 'Symptomless' spikes showed a similarly diverse community composition. Relative abundance at the genus level showed that filamentous ascomycetes like Cladosporium, Parastagonospora and Alternaria (Dothideomycetes) represented more than 50\% of the fungal abundance. However, Cladosporium was slightly more abundant in the 'Symptomless' bracts and kernels. On the other hand, endophytic basidiomycete yeasts (Vishniacozyma and Sporobolomyces, Tremellomycetes) were also abundant, but present in considerably lower proportions (near 15\%) in the samples with no visible FHB infection ('Control' and 'Symptomless'). This diversity contrasts with the one observed in 'FHB-symptoms' spikes, where Fusarium spp. represented up to $90 \%$ of the total fungal abundance. Interestingly, Fusarium spp. were also present, but at a lower proportion in the 'Symptomless' spikes, where they did not exceed $5 \%$ of the total relative abundance (Fig. 2a). 


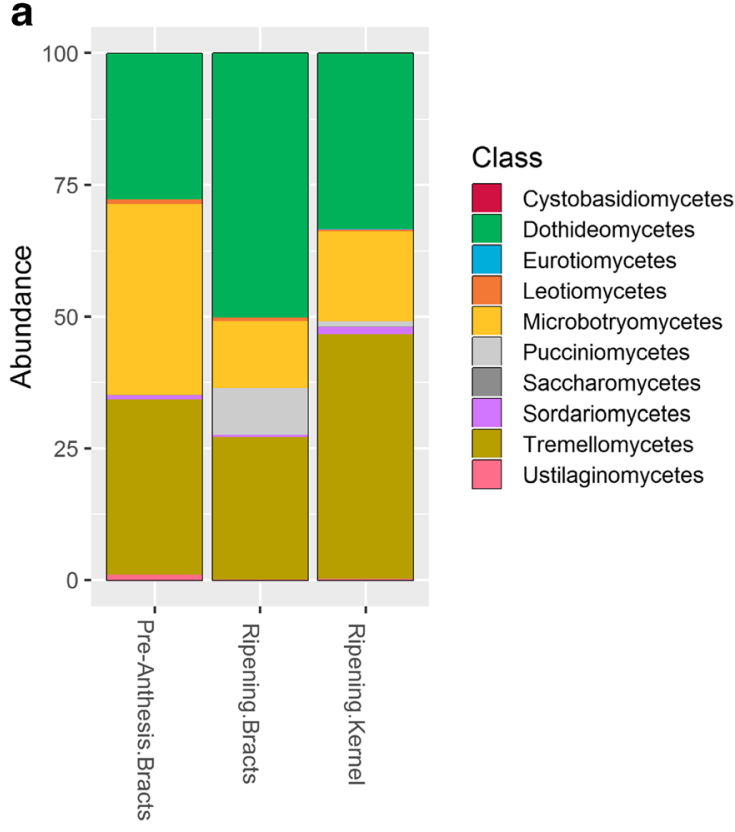

Fig. 1 Endophytic fungal community composition in wheat external bracts ('Bracts') and 'Kernels' before and after anthesis. a Relative abundance of the top 10 most abundant fungal classes in wheat heads at 'Pre-anthesis' and 'Ripening'. The reduction in relative abundance of Microbotryomycetes endophytic yeasts (yellow) at the 'Ripening' stage spikes is associated with an increase in Dothideomycetes (filamentous

Increased relative abundance of Fusarium was associated with a reduced abundance of other ascomycete fungi such as Cladosporium, Alternaria as well as some basidiomycetes like Sporobolomyces and Vishniacozyma. This tendency was similar for both tissue types 'Kernels' and 'Bracts', but especially clear in the kernels where Fusarium abundance reached almost 95\%. Genera such as Alternaria, Botrytis and Parastagonospora were more abundant in the external bracts than in the kernel. On the contrary, genera such as Fusarium, Cladosporium and Vishniacozyma were more abundant in the kernels compared to the external bracts. Additionally, a small increase

Table 1 PERMANOVA test results on beta diversity for fungal community changes during anthesis

\begin{tabular}{llc}
\hline Factor & $R^{2}$ & $P$ value \\
\hline Flowering stage $^{\mathrm{a}}$ & 0.046 & 0.007 \\
Tissue type $^{\mathrm{b}}$ & 0.073 & $<0.01$ \\
Location-cultivar $^{\mathrm{c}}$ & 0.089 & $<0.0001$ \\
Flowering stage $\times$ location-cultivar & 0.085 & $<0.0001$ \\
\hline
\end{tabular}

Effect of 'Location' and 'Cultivar' cannot be distinguished

${ }^{\text {a }}$ Flowering stage (Pre-Anthesis, Ripening)

${ }^{\mathrm{b}}$ Tissue type (Bracts, Kernels)

${ }^{\mathrm{c}}$ Location-cultivar (Sweden: cv. SW14038, Denmark: cv. KWS-Nils) ascomycetes) (green) in the external bracts and a similar increase in Tremellomycetes (basidiomycetes) in the kernels (ochre). b Alpha diversity (Observed, Shannon and Simpson) changes across the flowering stage and different tissues. 'Pre-anthesis' bracts showed significantly less Observed alpha diversity than 'Ripening' bracts $(* * P<0.01)$ using Wilcoxon signed-rank test

in the abundance of Botrytis was observed in 'FHB-symptoms' and 'Symptomless' spikes compared to the 'Control'. Overall, Fusarium infection was associated to a reduced abundance of most fungi inside wheat spikes.

Alpha diversity indices showed that 'FHB-symptoms' spikes had slightly less diversity than 'Symptomless' and 'Control' spikes. When looking at the interaction between 'Fusarium-status' and 'Tissue type', we observed that 'FHB symptoms' kernels showed lower alpha diversity than 'Control' and 'Symptomless' tissues $(P<0.05)$ (Fig. 2b). Similarly, we observed that in 'FHB-symptoms' spikes, fungal diversity was significantly lower during 2017 than in 2016 $(P<0.05)$ (Supplementary Fig. 3).

A PERMANOVA analysis showed that 'Fusarium-status' (FHB-symptoms, Symptomless or Control) accounted for most of the variation (12\%), with a high significance $\left(R^{2}=0.12, P<0.001\right)$. Similarly, 'Tissue type' (Bracts, Kernel) with $3 \%\left(R^{2}=0.03, P<0.001\right)$, 'Year' (2016, $2017)$ with $6 \%\left(R^{2}=0.06, P<0.001\right)$ and 'LocationCultivar' (Sweden: SW14308, Denmark: KWS-Nils) had a considerable effect $\left(R^{2}=0.01, P<0.001\right)$. Multiple interactions between factors were also detected in the community structure (Table 2). A principal coordinates analysis, based on Bray-Curtis dissimilarity of beta diversity, showed that 'FHB-symptoms' and 'Year' were the main two main drivers of the variation in the samples (Fig. 2c). In general, FHB symptoms were associated with a 

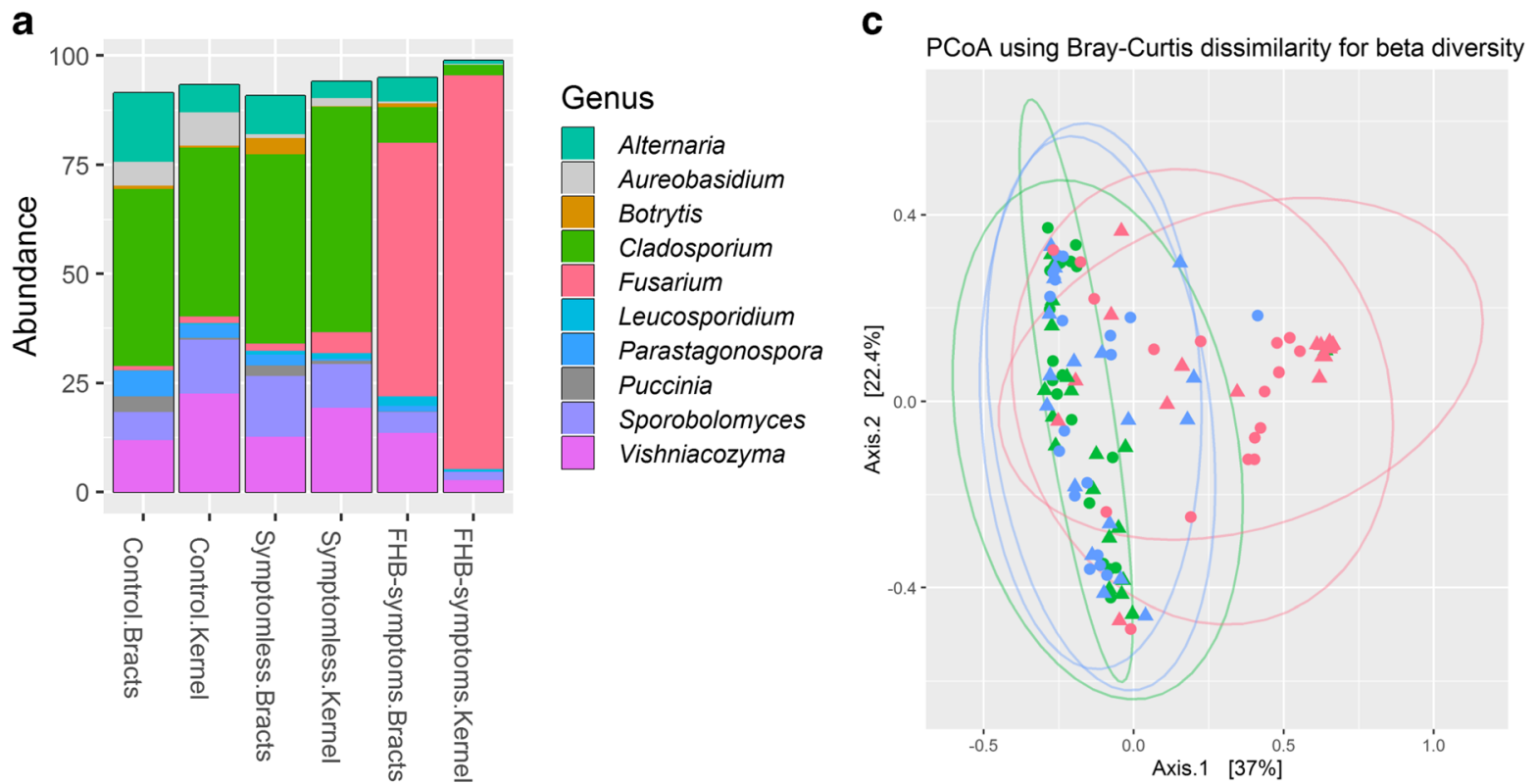

b
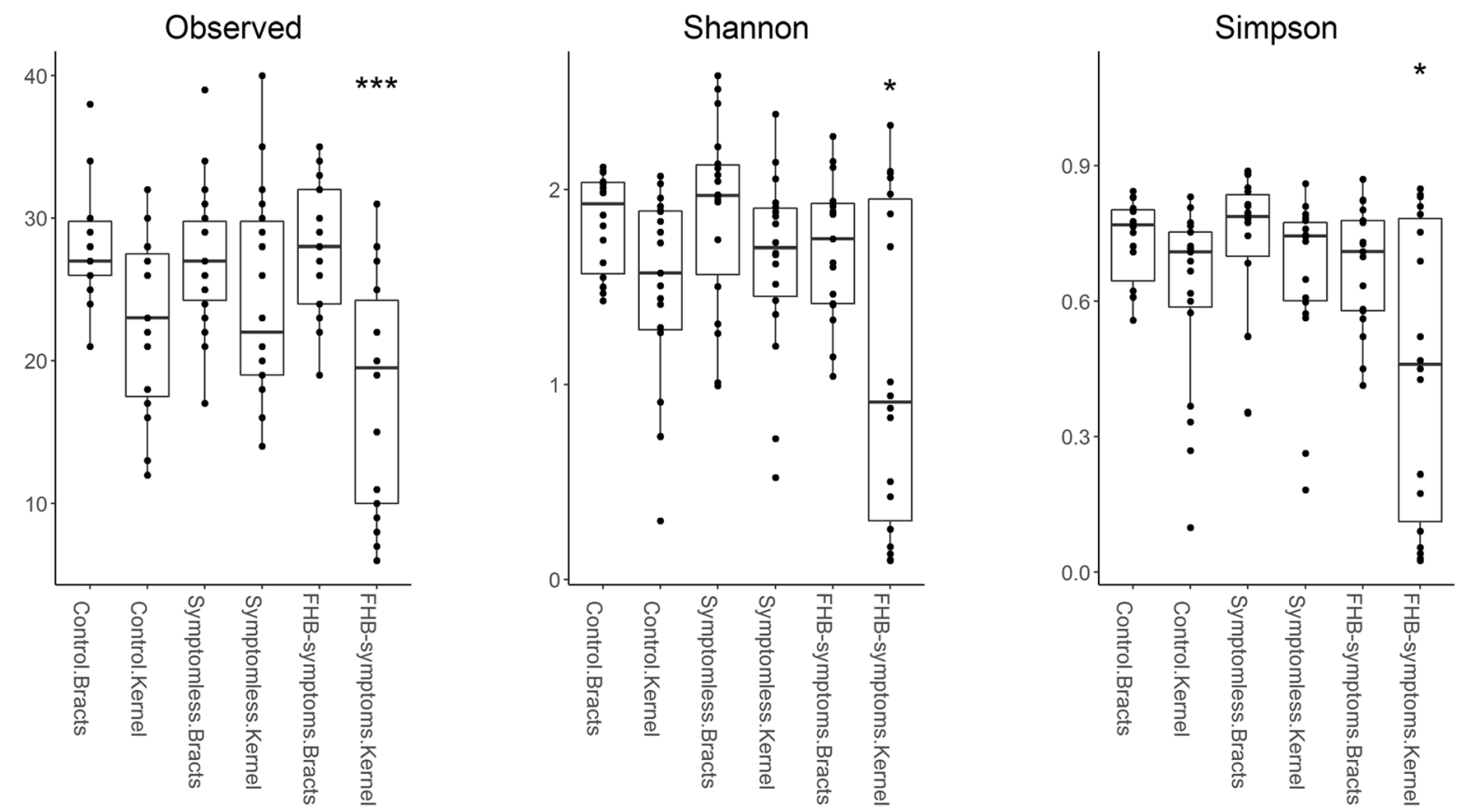

Fig. 2 Endophytic fungal community composition in wheat external bracts ('Bracts') and 'Kernels' during FHB attack. a Relative abundance of the top 10 most abundant fungal genera in wheat spikes from different groups: 'FHB-symptoms', 'Symptomless' and 'Control' (not inoculated). Fusarium infection is associated with changes in the community structure in 'FHB-symptoms' spikes and was correlated to reduced abundance of other fungi, especially in 'Kernels'. Cladosporium

and Vishniacozyma were the most abundant genera in 'Control' and 'Symptomless' spikes. b Alpha diversity (Observed, Shannon, Simpson) for each condition and tissue. 'FHB-symptoms' kernels showed less diversity $(* P<0.05, * * * P<0.001)$ using Kruskal-Wallis test. c PCoA using Bray-Curtis dissimilarity for beta diversity during FHB attack. 'FHB-symptoms' spikes clustered independently from other samples. Ellipses represent $95 \%$ confidence intervals

reduction in fungal species diversity in the spikes, especially inside infected kernels.

\section{Indicator Species of Different Communities}

An indicator species test was performed comparing Fusarium-status communities (FHB-symptoms,

Symptomless and Control) to determine key species in each group. The top 3 OTUs in each comparison of indicator species test are presented in Table 3. As expected, Fusarium spp. were the dominant species in the 'FHBsymptoms' spikes. At least 13 different OTUs belonging to the genus Fusarium were strong indicators of the spikes with symptoms when compared to the 'Control' 
Table 2 PERMANOVA test results on beta diversity for fungal community changes during FHB

\begin{tabular}{lll}
\hline Factor & $R^{2}$ & $P$ value \\
\hline Fusarium-status $^{\mathrm{a}}$ & 0.119 & $<0.0001$ \\
Tissue $^{\mathrm{b}}$ & 0.030 & $<0.0001$ \\
Year $^{\mathrm{c}}$ & 0.068 & $<0.0001$ \\
Location-cultivar $^{\mathrm{d}}$ & 0.019 & $<0.0001$ \\
Fusarium $_{\text {-status }} \times$ year & 0.082 & $<0.0001$ \\
Fusarium-status $\times$ location & 0.0046 & $<0.0001$
\end{tabular}

Effect of 'Location' and 'Cultivar' cannot be distinguished

${ }^{\text {a }}$ Fusarium-status (FHB-symptoms, Symptomless, Control)

${ }^{\mathrm{b}}$ Tissue type (Bracts, Kernels)

${ }^{c}$ Year $(2016,2017)$

${ }^{\mathrm{d}}$ Location-cultivar (Sweden: cv. SW14038, Denmark: cv. KWS-Nils)

and the 'Symptomless' spikes. Similarly, when assessing the indicator species of the 'Control' spikes against 'FHBsymptoms', only Cladosporium herbarum (OTU_2) emerged as an indicator of 'Control' spikes. Moreover, we observed that $C$. herbarum (OTU_2) was present in both 'Bracts' and 'Kernels', but it was more abundant in the 'Kernels' similar to Fusarium graminearum (OTU_1) (Fig. 3). Interestingly, within the 'Symptomless' spikes, different OTUs such as Itersonilia pannonica and Holtermanniella takashimae were strong indicators compared to 'FHB-symptoms'. Holtermanniella takashimae was also a strong indicator species when comparing 'Symptomless' and 'Control' spikes. These two OTUs were less abundant and more frequently found in the external bracts.

\section{Fungal OTUs Interactions Inside Wheat Spikes}

Co-occurrence analysis was used to detect specific interactions between OTUs in the communities. Most of the different OTUs positively correlated within their own genus. This was the case for the genera Fusarium (pink cluster), Vishniacozyma (purple cluster), Cladosporium (dark green cluster) and Alternaria (light blue cluster) (Fig. 4). Positive correlations across genera were observed between Cladosporium herbarum (OTU_2) and Alternaria infectoria (OTU_6). Interestingly, C. herbarum (OTU_2) also negatively correlated with three Fusarium graminearum OTUs, including the most abundant, F. graminearum (OTU_1). These negative correlations were observed both in the 'Kernels' and in the 'Bracts'. The correlations with their respective $P$ values can be seen in Supplementary Table 5 .

\section{Discussion}

A plethora of fungal species live unseen within plants tissues. These organisms, either alone or as communities, are believed to play an important role in plant fitness and therefore in crop performance. Understanding the way these endophytic communities respond to disease could broaden our knowledge of plant pathogens and pave the way for potential new disease control strategies based on biological control. To the best of our knowledge, this study represents the first analysis of fungal communities in wheat spikes under Fusarium head blight attack. Moreover, this is the first report of endophytic communities in wheat since previous studies have not attempted to distinguish between epiphytes and endophytes in wheat tissues. Using sodium hypochlorite surface-sterilized tissues, we
Table 3 Dufrene-Legendre Indicator Species test from each of the Fusarium status population comparisons

\begin{tabular}{lllll}
\hline Comparison & Fusarium-status & OTU & Indicator value & Closest BLAST hit \\
\hline 'FHB-Symptoms' & 'FHB-Symptoms' & OTU_1 & 0.87 & Fusarium graminearum \\
- 'Symptomless' & & OTU_396 & 0.69 & Fusarium culmorum \\
& & OTU_494 & 0.63 & Fusarium culmorum \\
& 'Symptomless' & OTU_2 & 0.72 & Cladosporium herbarum \\
& & OTU_377 & 0.30 & Holtermanniella takashimae \\
& & OTU_79 & 0.25 & Itersonilia pannonica \\
FHB-Symptoms & 'FHB-Symptoms' & OTU_399 & 0.92 & Fusarium culmorum \\
- Control & & OTU_1 & 0.91 & Fusarium graminearum \\
& & OTU_396 & 0.79 & Fusarium culmorum \\
Symptomless & Control & OTU_2 & 0.71 & Cladosporium herbarum \\
- Control & & OTU_11 & 0.77 & Leucosporidium fragarium \\
& & OTU_45 & 0.59 & Holtermanniella takashimae \\
& & OTU_377 & 0.30 & Holtermanniella takashimae \\
& Control & No species detected & \\
\hline
\end{tabular}

${ }^{\text {a }}$ Fusarium-status (spikes with FHB-symptoms, Symptomless and Control) 

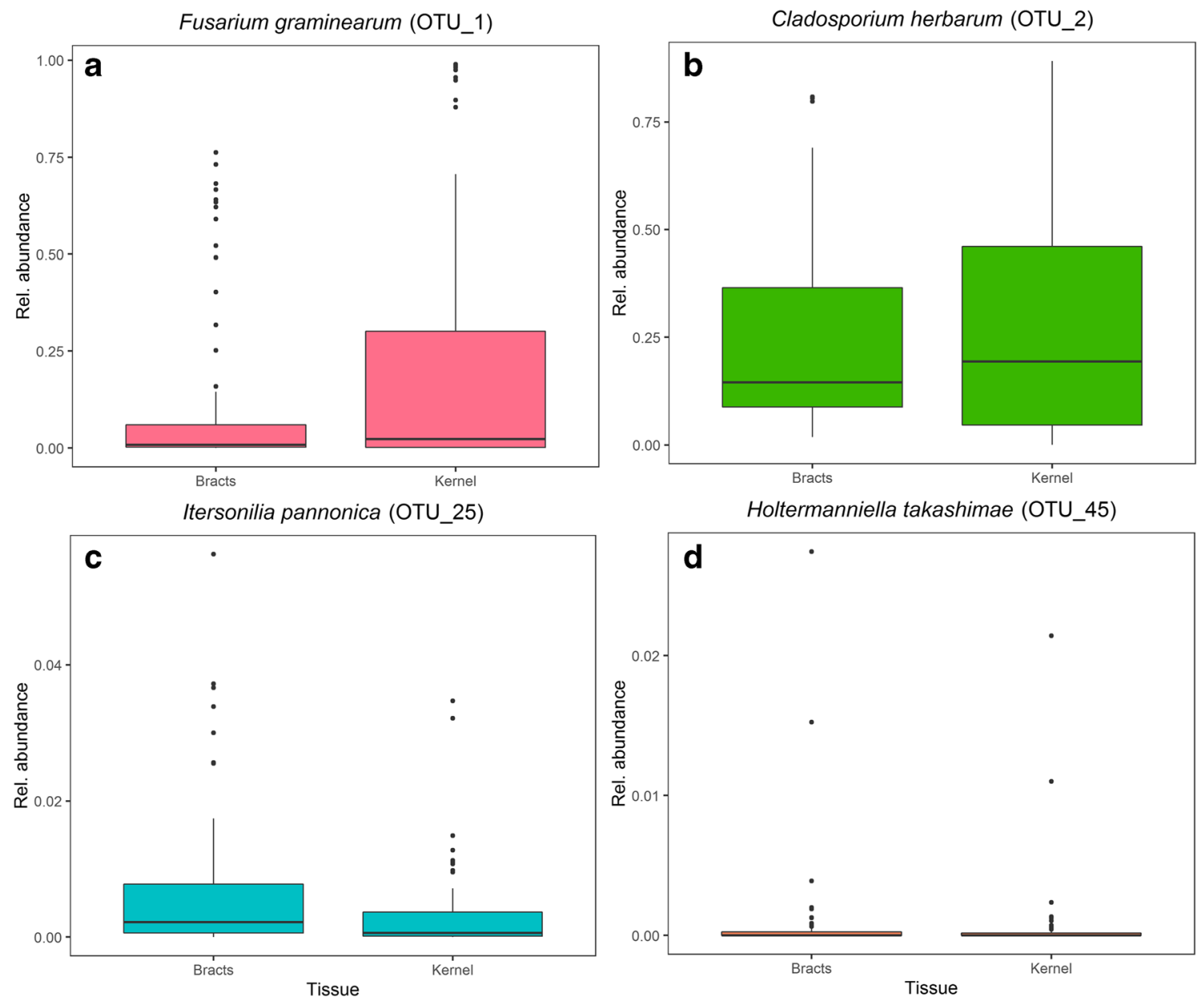

Fig. 3 Relative abundance of four different fungal OTUs inside wheat spike tissues. a Fusarium graminearum (OTU 1) was detected in higher abundance in kernels. b Cladosporium herbarum (OTU_2) was observed in higher proportion in kernels than in the external bracts. c Itersonilia

pannonica (OTU_25) was more abundant in the 'Bracts' than in the 'Kernels'. d Holtermanniela takashimae (OTU 45) showed low abundance both in 'Kernels' and 'Bracts'. Only C. herbarum appear to compete for the same biological niche as the pathogens

Fig. 4 Network plot for cooccurrence between different OTUs identified in wheat spikes in this study. Positive correlations are shown as blue edges and negative correlations as red edges between different OTUs (nods). OTUs from the same genera are visualized with the same colour. Four fungal genera interacted closely with each other: Fusarium (pink), Alternaria (light blue), Vishniacozyma (purple) and Cladosporium (dark green) Cladosporium herbarum (OTU 2) negatively correlated with three Fusarium graminearum OTUs
- Alternaria

- Anthracocystis

- Ascochyta

- Cladosporium

- Didymella

- Dioszegia

- Filobasidium

- Fusarium

- Itersonilia

- Neoascochyta

- Parastagonospora

- Sarocladium

- Sporobolomyces

- Vishniacozyma

- Cladosporium

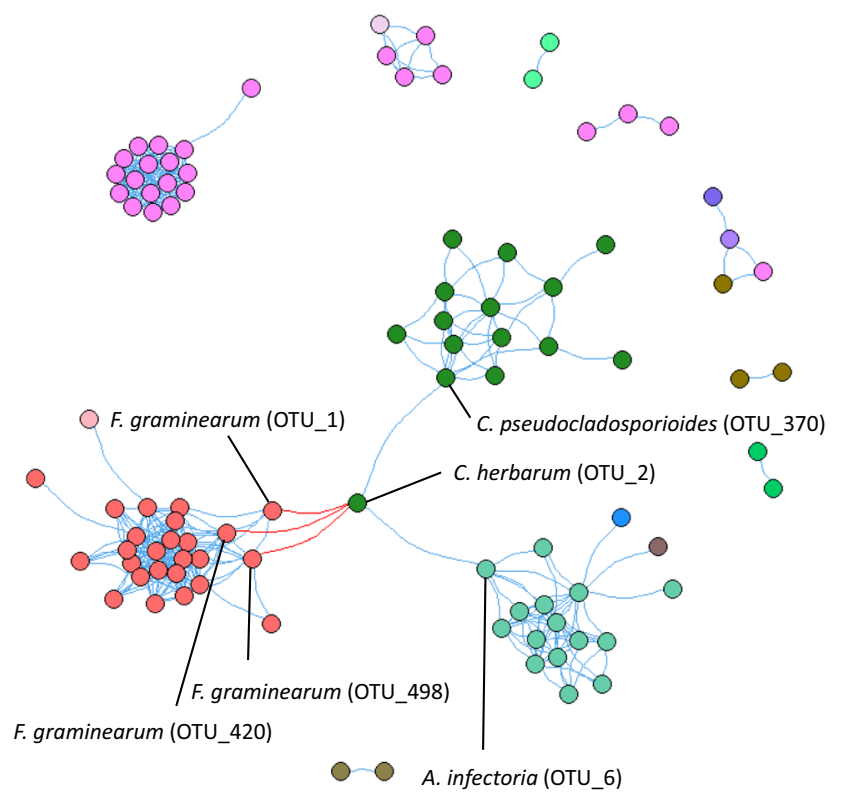


enriched the samples with endophytic fungi in the analysis by removing most of the external microbiota [33].

We observed how closely associated endophytic communities inside wheat spikes tend to increase in diversity as anthesis occurs and kernel develop. Additionally, we identified a succession from a basidiomycete yeast-dominated community before anthesis to a filamentous ascomycete-rich community during ripening. Most importantly, we showed how Fusarium infection interacts with these dynamics. The pathogens quickly colonize tissues and are associated with a reduction in fungal diversity, especially inside the newly formed kernels.

Fungal communities in wheat tissues (root, crowns, tillers, leaves, glumes, awns and anthers) have been assessed using culture-dependent methods in the past [9, 34, 35]. These approaches have only examined the limited cultivable communities selected by the isolation media. Most of these studies have identified between 30 and 50 fungal species and have shown minor representation of phylum Basidiomycota (1$2 \%$ ) $[34,35]$. On the other hand, most culture-independent studies, including this one, have detected higher numbers of species and have observed that basidiomycete diversity constitutes nearly $25 \%$ of the total fungal species [36-38], but this can change depending on the ITS amplification primers used [39]. In general, culture-dependent methods have been shown to isolate and identify the main players in the wheat mycobiome but the resolution to detect variations and specific interactions remains low. Culture-independent methods provide a better image of the communities and their dynamics.

Our data reveal that changes in fungal communities during flowering are quantitative rather than qualitative. We observed a reduction in abundance of Microbotryomycetes yeasts (Sporobolomyces and Leucosporidium) at the ripening stage. This reduction was associated with an increase in abundance of Tremellomycetes yeasts (Vishniacozyma, Cryptococcus, Itersonilia, Dioszegia) in the 'Kernels' and Dothideomycetes (largest group of ascomycota) in the 'Bracts'.

Similar dynamics have also been observed in fungal communities on wheat leaves and stems (including epiphytes). Dothideomycete species increased in abundance while Microbotryomycetes and Tremellomycete species were reduced from pre-flowering (BBCH 45) to seed development (BBCH 85) [37, 38]. For wheat spikes, it has been observed that the ratio of ascomycetes to basidiomycetes increased during kernel development [36]. Dothideomycete species such as Cladosporium cladiosporoides, Alternaria infectoria and Parastagonospora avenae were the most abundant of these fungi in our study. These have been reported as pathogens in other cereals, but most commonly as later stage saprophytes on wheat. Other studies have demonstrated that wheat spikes and grains harbour a high diversity of these ascomycetes [36, 39, 40]. Similar trends have been observed in wheat leaves as Zymoseptoria tritici was found to be the most abundant fungus in later stage leaves [23, 41]. This suggests a general increase in abundance of opportunistic or even pathogenic fungi over time in wheat tissues. Our data indicate that this shift in fungal communities also occurs inside wheat spikes and that it commences during anthesis, earlier than previously reported. Interestingly, we showed that ascomycete enrichment was not detectable in wheat kernels. This suggests that fungal diversity in external tissues such as glumes and lemmas ('Bracts') increase over time as they are environmentally exposed to airborne spores while the kernels appear to be protected from these changes, at least during early ripening.

Using two methods of inoculation during the flowering stage, it was possible to measure the effect of FHB on fungal communities in wheat head tissues. The Fusarium isolates that were used for inoculation rapidly colonized the tissues and established in the community. The fungi caused visible disease at 14 days after inoculation and this was coupled to significant changes in the fungal community. Increasing abundance of Fusarium (above 80\%) in the infected spikes was associated with a reduced abundance of other fungi, representing both ascomycetes and basidiomycetes. This process was similar for both 'Kernels' and 'Bracts', but was especially noticeable in the 'Kernels'.

A few studies have assessed the effect of pathogens on established microbial communities in plant tissue. Evaluation of the fungal diversity of wheat leaves showing symptoms of Stagonospora nodorum blotch reported the co-existence of several pathogens in these symptomatic leaves, but there was no comparison against healthy leaves [42]. Recently, it was shown that the most abundant endophytic bacterial genera in Fusarium-infected wheat spikes increased in relative abundance (from 2 to 10 fold) compared to healthy spikes under field conditions [43]. Similarly, bacterial diversity increased in Paullinia cupana leaves affected by anthracnose symptoms (Colletotrichum spp.) compared to leaves with no symptoms [44]. A similar tendency was detected, although not statistically significant, for bacterial communities in tomato roots attacked by the nematode Meloidogyne incognita when compared to non-infected roots [45].

Opportunistic bacteria can benefit from pathogen attack, but in the case of fungi, our data support the hypothesis that the Fusarium spp. either prevent other fungi from establishing and growing through direct competition inside wheat spikes and/or they hinder the growth of the rest of the community by modifying the environment inside the tissue to their favour. The Fusarium-wheat molecular interaction has been described to occur in two phases: a first phase (asymptomatic) where the pathogen metabolism focuses on plant-defence suppression and growth and a second phase where nutrient depletion activates the production of cell-degrading enzymes [3]. Likewise, production of mycotoxins, specifically deoxynivalenol (DON), has 
been observed during the early stage [46]. Interestingly, it has been shown that Fusarium infection induces production of putrescine, a DON promoter, in the wheat spikes [47]. This suggests an active role of the pathogen in changing the internal chemical conditions of the spike during infection. Indeed, the known role of specialized metabolites, such as the mycotoxins DON and zearalenone, is consistent with their ability to outcompete other fungi $[48,49]$. We observed that diversity was drastically reduced in the 'Kernels' while remaining similar or slightly higher in the 'Bracts' during symptomatic infection. Symptomless spikes (which showed low levels of Fusarium abundance) did not show such a reduction. This suggests that the reduction in endophyte diversity associated to Fusarium infection is likely to occur during the second phase of infection and is mediated by the physiological changes caused by the production of enzymes and mycotoxins in the kernel.

Finally, one of the objectives of this study was to identify potentially naturally occurring endophytic fungi associated with healthy spikes. We collected spikes that remained visually healthy after pathogen exposure ('Symptomless') and compared their endophytic communities to the ones in spikes with symptoms 'FHB-symptoms'. A prospective candidate would theoretically be enriched in 'Symptomless' spikes and/or negatively correlated with Fusarium spp. We identified Cladosporium herbarum, Holtermanniella takashimae and Itersonilia pannonica as indicator species of the 'Symptomless' spikes. Additionally, we detected a strong negative correlation between Cladosporium herbarum and three Fusarium graminearum OTUs.

Cladosporium herbarum is a common endophyte and an environmentally ubiquitous saprophytic fungus [50]. It has been associated with cereals and isolated from wheat tissues in several studies [9] and has even been reported to be pathogenic in some wheat cultivars [51]. We found higher C. herbarum abundance in 'Kernels', similar to Fusarium spp. The observed negative correlation between these two species suggests that $C$. herbarum and $F$. graminearum compete for the same biological niche. It is worth noting that we found high $C$. herbarum abundance in spikes with no visible symptoms. Similar negative interactions with Fusarium spp. have been observed for other ascomycete fungi such as Parastagonospora nodorum in grains and tillers [38]. However, this was seen in a study where Fusarium infection was not recorded. Interestingly, several studies have tested Cladosporium spp. as biocontrol agents. Cladosporium cladosporioides and C. pseudocladosporioides were shown to have antagonistic effects against Puccinia horiana, a pathogen in chrysanthemum [52]. However, Cladosporium halotolerans has been tested against $F$. graminearum in wheat with little success [53].
On the other hand, Holtermanniella takashimae, Itersonilia pannonica and other tremellomycetous yeasts have been found to be ubiquitous colonizers of plant tissues [54]. These two species were detected in both the 'Kernels' and the 'Bracts', but they were most abundant in the 'Bracts'. This means that they do not necessarily compete with Fusarium spp. for the same niche, but could rather prevent Fusarium infection as they appear to be enriched in healthy plants. Tremellomycete yeasts, including the genus Cryptococcus, have been shown to provide growth enhancement to plants [55]. In fact, the yeast isolate Cryptococcus flavescens, strain $\mathrm{OH}$ 182.9, isolated from wheat anthers, reduced Fusarium disease severity and mycotoxin accumulation in wheat and furthermore increased kernel weight compared to the infected control [56, 57]. This isolate was also observed to be able to colonize the abaxial surfaces of glume and lemma tissues [58], the same tissues where we observed them in higher abundance. Whether C. herbarum, H. takashimae or I. pannonica outcompete or prevent $F$. graminearum colonization of wheat spikes remains to be determined. However, our data suggest they could potentially be involved in a naturally occurring biocontrol effect against FHB.

The new microbiome perspective to plant-microbe interactions allows a more holistic approach to plant disease control and opens the door to unexplored ways of biological control. FHB results in an imbalance on the fungal communities in wheat spikes. Theoretically, it will be possible to modify the wheat microbiome with beneficial microorganisms that counterbalance the specialized mechanisms of pathogens to maintain system homeostasis. Future work must aim to identify more of these interactions in natural FHB-infested areas.

Acknowledgements We would like to thank Helena Applegren (Svalöv) for her guidance during field sampling and Fusarium disease scoring as well as the helpful people at both Flakkebjerg and Svalöv field stations, technician Ulla Rasmussen for her dedicated work in the lab, as well as Helle Sørensen and Bo Markussen from Department of Mathematical Sciences at Copenhagen University for their feedback on the statistical analyses.

Funding Information This project has received funding from the European Union's Horizon 2020 research and innovation programme under the Marie Skłodowska-Curie grant agreement No. 674964.

\section{Compliance with Ethical Standards}

Conflict of Interest The authors declare no conflict of interest.

Open Access This article is distributed under the terms of the Creative Commons Attribution 4.0 International License (http:// creativecommons.org/licenses/by/4.0/), which permits unrestricted use, distribution, and reproduction in any medium, provided you give appropriate credit to the original author(s) and the source, provide a link to the Creative Commons license, and indicate if changes were made. 


\section{References}

1. Food and Agriculture Organization of the United Nations (2017) FAOSTAT Database. FAO, Rome, Italy Retrieved 19 November 2018 from http://faostat3.fao.org/home/E

2. Figueroa M, Hammond-Kosack KE, Solomon PS (2018) A review of wheat diseases - a field perspective. Mol Plant Pathol 19:15231536. https://doi.org/10.1111/mpp.12618

3. Rojas EC, Jørgensen HJL, Jensen B, Collinge DB (2018) Fusarium diseases: biology and management perspectives. In: Oliver RP (ed) Integrated disease management of wheat and barley. Burleigh Dodds Science Publishing, Cambridge. https://doi.org/10.19103/ AS.2018.0039.02

4. Nielsen LK, Jensen JD, Nielsen GC et al (2011) Fusarium head blight of cereals in Denmark: species complex and related mycotoxins. Phytopathology 101:960-969. https://doi.org/10.1094/ PHYTO-07-10-0188

5. Pasquali M, Beyer M, Logrieco A et al (2016) A European database of Fusarium graminearum and F. culmorum trichothecene genotypes. Front Microbiol 7. https://doi.org/10.3489/fmicb.2016. 00406

6. Boenisch MJ, Schäfer W (2011) Fusarium graminearum forms mycotoxin producing infection structures on wheat. BMC Plant Biol 11:110. https://doi.org/10.1186/1471-2229-11-110

7. Lee HJ, Ryu D (2017) Worldwide occurrence of mycotoxins in cereals and cereal-derived food products: public health perspectives of their co-occurrence. J Agric Food Chem 65:7034-7051. https:// doi.org/10.1021/acs.jafc.6b04847

8. Ma LJ, Geiser D, Proctor R, Rooney A, O'Donell K, Trail F, Gardiner D, Manners J, Kazan K (2013) Fusarium pathogenomics. Annu Rev Microbiol 67:399-416. https://doi.org/10.1146/annurevmicro-092412-155650

9. Döll S, Dänicke S (2011) The Fusarium toxins deoxynivalenol $(\mathrm{DON})$ and zearalenone (ZON) in animal feeding. Prev Vet Med 102:132-145. https://doi.org/10.1016/j.prevetmed.2011.04.008

10. Comby M, Lacoste S, Baillieul F, Profizi C, Dupont J (2016) Spatial and temporal variation of cultivable communities of cooccurring endophytes and pathogens in wheat. Front Microbiol 7. https://doi.org/10.3389/fmicb.2016.00403

11. Card S, Johnson L, Teasdale S, Caradus J (2016) Deciphering endophyte behaviour: the link between endophyte biology and efficacious biological control agents. FEMS Microbiol Ecol 92:fiw114. https://doi.org/10.1093/femsec/fiw114

12. Berg G, Rybakova D, Grube M, Köberl M (2016) The plant microbiome explored: implications for experimental botany. J Exp Bot 67:995-1002. https://doi.org/10.1093/jxb/erv466

13. Lata R, Chowdhury S, Gond SK, White JF (2018) Induction of abiotic stress tolerance in plants by endophytic microbes. Lett Appl Microbiol 66:268-276. https://doi.org/10.1111/lam.12855

14. Murphy BR, Martin Nieto L, Doohan FM, Hodkinson TR (2015) Fungal endophytes enhance agronomically important traits in severely drought-stressed barley. J Agron Crop Sci 201:419-427. https://doi.org/10.1111/jac.12139

15. Rabiey M, Shaw MW (2016) Piriformospora indica reduces fusarium head blight disease severity and mycotoxin DON contamination in wheat under UK weather conditions. Plant Pathol 65:940952. https://doi.org/10.1111/ppa.12483

16. Busby PE, Ridout M, Newcombe G (2016) Fungal endophytes: modifiers of plant disease. Plant Mol Biol 90:645-655. https://doi. org/10.1007/s11103-015-0412-0

17. Collinge DB, Jørgensen HJL, Latz M et al (2018) Searching for novel fungal biological control agents for plant disease control among endophytes. In: Hodkinson T, Doohan F, Saunders M, Murphy B (eds) Endophytes for a growing world. Cambridge Univ Press
18. Latz M, Jensen B, Collinge DB, Jørgensen HJL (2019) Endophytic fungi as biocontrol agents: elucidating mechanisms in disease suppression. Plant Ecol Divers. https://doi.org/10.1080/17550874. 2018.1534146

19. Lancashire $P$, Bleiholder $H$, van den Boom T et al (1991) A uniform decimal code for growth stages of crops and weeds. Ann Appl Biol 1119:561-601. https://doi.org/10.1111/j.1744-7348.1991.tb04895. $\mathrm{X}$

20. Ihrmark K, Bödeker ITM, Cruz-Martinez K et al (2012) New primers to amplify the fungal ITS2 region - evaluation by 454 sequencing of artificial and natural communities. FEMS Microbiol Ecol 82:666-677. https://doi.org/10.1111/j.1574-6941. 2012.01437.x

21. Wu L, Wen C, Qin Y et al (2015) Phasing amplicon sequencing on Illumina Miseq for robust environmental microbial community analysis. BMC Microbiol 15:1-12. https://doi.org/10.1186/ s12866-015-0450-4

22. Unterseher M, Siddique AB, Brachmann A, Peršoh D (2016) Diversity and composition of the leaf mycobiome of beech (Fagus sylvatica) are affected by local habitat conditions and leaf biochemistry. PLoS One 11:1-16. https://doi.org/10.1371/journal. pone. 0152878

23. Sapkota R, Jørgensen LN, Nicolaisen M (2017) Spatiotemporal variation and networks in the mycobiome of the wheat canopy. Front Plant Sci 8:1-10. https://doi.org/10.3389/fpls.2017.01357

24. Caporaso JG, Kuczynski J, Stombaugh J et al (2010) QIIME allows analysis of highthroughput community sequencing data. Nat Methods 7:335-336. https://doi.org/10.1038/nmeth.f.303

25. Aronesty E (2013) Comparison of sequencing utility programs. Open Bioinforma J 7:1-8. https://doi.org/10.2174/ 1875036201307010001

26. Rognes T, Flouri T, Nichols B, Quince C, Mahé F (2016) VSEARCH: a versatile open source tool for metagenomics. PeerJ 4:e2584. https://doi.org/10.7717/peerj.2584

27. Abarenkov K, Nilsson RH, Larsson KH et al (2010) The UNITE database for molecular identification of fungi-recent updates and future perspectives. New Phytol 186:281-285. https://doi.org/10. $1111 /$ j.1469-8137.2009.03160.x

28. R Core Team (2013) R: a language and environment for statistical computing. R Foundation for statistical computing, Vienna, Austria URL http://www.R-project.org/

29. Oksanen A, Blanchet FG, Friendly M, et al. (2017) R package · vegan ' [software]. https://cran.r-project.org/web/packages/vegan/ index.html

30. McMurdie PJ, Holmes S (2013) Phyloseq: an R package for reproducible interactive analysis and graphics of microbiome census data. PLoS One 8. https://doi.org/10.1371/journal.pone.0061217

31. Roberts D. (2016) labdsv: ordination and multivariate analysis for ecology. https://cran.r-project.org/package=labdsv

32. Gabor C, Nepusz T (2006) The igraph software package for complex network research. InterJournal.:1695 http://igraph.org

33. Kampmann ML, Børsting C, Morling N (2017) Decrease DNA contamination in the laboratories. Forensic Sci Int Genet Suppl Ser 6:e577-e578. https://doi.org/10.1016/j.fsigss.2017.09.223

34. Sieber T, Riensen K, Müller E, Fried PM (1988) Endophytic fungi in four winter wheat cultivars (Triticum aestivum) differing in resistance against stagonospora. J Phytopathol 122:289-306

35. Vujanovic V, Mavragani D, Hamel C (2012) Fungal communities associated with durum wheat production system: a characterization by growth stage, plant organ and preceding crop. Crop Prot 37:2634. https://doi.org/10.1016/j.cropro.2012.02.006

36. Hertz M, Jensen IR, Jensen LØ et al (2016) The fungal community changes over time in developing wheat heads. Int J Food Microbiol 222:30-39. https://doi.org/10.1016/j.ijfoodmicro.2016.01.018

37. Gdanetz K, Trail F (2017) The wheat microbiome under four management strategies, and potential for endophytes in disease 
protection. Phytobiomes. 1:158-168. https://doi.org/10.1094/ PBIOMES-05-17-0023-R

38. Grudzinska-Sterno M, Yuen J, Stenlid J, Djurle A (2016) Fungal communities in organically grown winter wheat affected by plant organ and development stage. Eur J Plant Pathol 146:401-417. https://doi.org/10.1007/s10658-016-0927-5

39. Nicolaisen M, Justesen AF, Knorr K, Wang J, Pinnschmidt HO (2014) Fungal communities in wheat grain show significant coexistence patterns among species. Fungal Ecol 11:145-153. https://doi.org/10.1016/j.funeco.2014.06.002

40. Shi Y, Cheng Y, Wang Y et al (2017) Investigation of the fungal community structures of imported wheat using highthroughput sequencing technology. PLoS One 12:1-10. https://doi.org/10.1371/ journal.pone. 0171894

41. Karlsson I, Friberg H, Kolseth AK, Steinberg C, Persson P (2017) Organic farming increases richness of fungal taxa in the wheat phyllosphere. Mol Ecol 26:3424-3436. https://doi.org/10.1111/ mec. 14132

42. Blixt E, Olson Å, Lindahl B, Djurle A, Yuen J (2010) Spatiotemporal variation in the fungal community associated with wheat leaves showing symptoms similar to stagonospora nodorum blotch. Eur J Plant Pathol 126:373-386. https://doi.org/10.1007/ s10658-009-9542-Z

43. Chen Y, Wang J, Yang N et al (2018) Wheat microbiome bacteria can reduce virulence of a plant pathogenic fungus by altering histone acetylation. Nat Commun 9:3429. https://doi.org/10.1038/ s41467-018-05683-7

44. Bogas AC, Ferreira AJ, Araújo WL et al (2015) Endophytic bacterial diversity in the phyllosphere of Amazon Paullinia cupana associated with asymptomatic and symptomatic anthracnose. Springerplus. 4:1-13. https://doi.org/10.1186/s40064-015-1037-0

45. Tian BY, Cao Y, Zhang KQ (2015) Metagenomic insights into communities, functions of endophytes, and their associates with infection by root-knot nematode, Meloidogyne incognita, in tomato roots. Sci Rep 5:1-15. https://doi.org/10.1038/srep17087

46. Gardiner D, Kazan K, Manners J (2009) Novel genes of Fusarium graminearum that negatively regulate deoxynivalenol production and virulence. Mol Plant-Microbe Interact 22:1588-1600

47. Gardiner D, Kazan K, Praud S, Torney F, Rusu A, Manners J (2010) Early activation of wheat polyamine biosynthesis during Fusarium head blight implicates putrescine as an inducer of trichothecene mycotoxin production. BMC Plant Biol 10:289

48. Lutz M, Ferchtinger G, Défago G, Duffy B (2003) Mycotoxigenic Fusarium and deoxynivalenol production repress chitinase gene expression in the biocontrol agent Trichoderma atroviride P1. App Env Microbiol 69:3077-3084. https://doi.org/10.1128/AEM. 69.6.3077-3084.2003
49. Kosawang C, Karlsson M, Vélez H, Rasmussen P, Collinge D, Jensen B, Jensen D (2014) Zearalenone detoxification by zearalenon hydrolase is important for the antagonistic ability of Clonostachys rosea against mycotoxigenic Fusarium graminearum. Fungal Biology 118:364-373. https://doi.org/10. 1016/j.funbio.2014.01.005

50. Schubert K, Groenewald JZ, Braun U et al (2007) Biodiversity in the Cladosporium herbarum complex (Davidiellaceae, Capnodiales), with standardisation of methods for Cladosporium taxonomy and diagnostics. Stud Mycol 58:105-156. https://doi. org $/ 10.3114 / \operatorname{sim} .2007 .58 .05$

51. Perelló A, Sisterna M (2008) Formation of Lewia infectoria, the teleomorph of Alternaria infectoria, on wheat in Argentina. Australas Plant Pathol 37:589-591. https://doi.org/10.1071/ AP08060

52. Torres DE, Rojas-Martínez RI, Zavaleta-Mejía E, Guevara-Fefer P, Márquez-Guzmán GJ, Pérez-Martínez C (2017) Cladosporium cladosporioides and Cladosporium pseudocladosporioides as potential new fungal antagonists of Puccinia horiana Henn., the causal agent of chrysanthemum white rust. PLoS One 12:1-16. https:// doi.org/10.1371/journal.pone.0170782

53. Comby M, Gacoin M, Robineau M et al (2017) Screening of wheat endophytes as biological control agents against Fusarium head blight using two different in vitro tests. Microbiol Res 202:11-20. https://doi.org/10.1016/j.micres.2017.04.014

54. Pitkäranta M, Meklin T, Hyvärinen A et al (2008) Analysis of fungal flora in indoor dust by ribosomal DNA sequence analysis, quantitative PCR, and culture. Appl Environ Microbiol 74:233244. https://doi.org/10.1128/AEM.00692-07

55. Mestre MC, Fontenla S, Bruzone MC, Fernández NV, Dames J (2016) Detection of plant growth enhancing features in psychrotolerant yeasts from Patagonia (Argentina). J Basic Microbiol 56:1098-1106. https://doi.org/10.1002/jobm.201500728

56. Khan NI, Schisler DA, Boehm MJ, Slininger PJ, Bothast RJ (2001) Selection and evaluation of microorganisms for biocontrol of fusarium head blight of wheat incited by Gibberella zeae. Plant Dis 85: 1253-1258. https://doi.org/10.1094/PDIS.2001.85.12.1253

57. Zhang S, Schisler DA, Boehm MJ, Slininger PJ (2007) Utilization of chemical inducers of resistance 2nd Cryptococcus flavescens $\mathrm{OH}$ 182.9 to reduce Fusarium head blight under greenhouse conditions. Biol Control 42:308-315. https://doi.org/10.1016/j.biocontrol. 2007.05.020

58. Schisler DA, Core AB, Boehm MJ et al (2014) Population dynamics of the Fusarium head blight biocontrol agent Cryptococcus flavescens $\mathrm{OH} 182.9$ on wheat anthers and heads. Biol Control 70:17-27. https://doi.org/10.1016/j.biocontrol.2013.11.011 